TMS-EEG indexes abnormal GABAergic signalling in patients with schizophrenia

Sukhwinder S Shergill ${ }^{\star}$, Viviana Santoro ${ }^{2}$, Lorenzo Rocchi ${ }^{3}$, Meng Di Hou ${ }^{1}$ and Isabella Premoli ${ }^{2}$

${ }^{1}$ Department of Psychosis Studies, Institute of Psychiatry, Psychology and Neuroscience (IoPPN), King's College London; ${ }^{2}$ Department of Basic and Clinical Neuroscience, Institute of Psychiatry, Psychology and Neuroscience, King's College London and ${ }^{3}$ Department of Clinical and Movement Neurosciences UCL Queen Square Institute of Neurology, University College London

${ }^{\star}$ Corresponding author.

doi: 10.1192/bjo.2021.185

Aims. Transcranial magnetic stimulation (TMS) is a non-invasive brain stimulation tool designed to probe the strength of inhibitory and excitatory neurotransmission in the cortex. Combined with electromyography, paired-pulse TMS paradigms have revealed a deficit in inhibition mediated by GABA-A receptors in patients with schizophrenia. Combined TMS-electroencephalography (TMSEEG) provides a more detailed examination of cortical excitability and may shed more light into the pathophysiology of schizophrenia. Of the various peaks of the TMS-evoked EEG signal, responses at 45 (N45) and $100 \mathrm{~ms}$ (N100) likely reflect GABA-A and GABA-B receptor-mediated inhibition, respectively. Responses at $25 \mathrm{~ms}$ (P25) are affected by voltage-gated channel ligands, whereas glutamatergic processes may be related to the P70 component. We here aim to systematically investigate the role of these neural processes in patients with schizophrenia by using TMS-EEG.

Method. TMS-evoked EEG potentials (TEPs) were recorded in patients with schizophrenia $(\mathrm{n}=19)$ and in age-matched healthy controls $(n=17) .150$ TMS pulses at $90 \%$ of resting motor threshold were applied over the left primary motor cortex during EEG recording. Differences in TEPs between the two groups were analysed for all electrodes and for time windows corresponding to each TEP (P25: 0.015-0.035 ms; N45: 0.035-0.06 ms; P70: 0.035-0.06 ms; N100: 0.09-0.14ms) by applying multiple independent sample t-tests. Further, a cluster-based permutation analysis approach was implemented to correct for multiple comparisons.

Result. Compared to controls, patients showed amplitude reduction for the P25 (negative and positive cluster; $\mathrm{p}<0.001$ and $\mathrm{p}=$ 0.04 , respectively), N45 (negative and positive cluster; $\mathrm{p}<0.001$ and $\mathrm{p}=0.001$, respectively) and P70 component (negative and positive cluster; $\mathrm{p}=0.04$ and $\mathrm{p}=0.004$, respectively).

Conclusion. There results extend on previous literature about impairment of GABA-A receptor mediated inhibition in schizophrenia, as demonstrated by the N45 amplitude reduction, whereas no significant differences in GABA-B index (i.e., N100) were revealed. Our results also showed that, although specific mechanisms underlying P25 and P70 have not been fully elucidated yet, excitatory neurotransmission is altered in this clinical population. To conclude, TMS-EEG may provide a more comprehensive view of the inhibitory and excitatory mechanisms involved in the pathophysiology of schizophrenia.

\section{Anxiety levels during COVID 19 pandemic in primary and secondary doctors in UK}

Dr Shweta Mittal ${ }^{1 \star}$ and Abdalla Abouebeid ${ }^{2}$

${ }^{1}$ Nottinghamshire Healthcare NHS Foundation Trust, Bassetlaw

Mental Health Services and ${ }^{2}$ The St.Vincent Practice

${ }^{*}$ Corresponding author.

doi: 10.1192/bjo.2021.186
Aims. The study aims to examine the severity of anxiety in primary and secondary doctors in the UK during first wave of COVID-19 pandemic.

Method. An online General Anxiety Disorder-7 (GAD7) survey was distributed during the first wave of COVID-19 pandemic (April-May 2020) to doctors in primary and secondary care in the UK. Seven closed-ended questions were included in the questionnaire. Respondents were to indicate how frequently they experienced specific issues in the previous fortnight: Feeling nervous, anxious, or on edge; being unable to stop or control worrying; worrying too much generally; trouble relaxing; being so restless that it's hard to sit still; becoming easily annoyed or irritable, feeling afraid of something awful happening. Participants were required to tick one of four choices for each of the seven parameters - not at all (0), several days (1), more than half the days (2) and nearly every day (3). A person with minimal or no anxiety will score less than 5 . The survey was anonymous and circulated in professional online doctors' forums. Participation was voluntary and no incentives were given.

Result. 273 completed surveys were received; 120 doctors were in primary care and 153 were in secondary care. Average GAD7 score was 6.4 in primary care and 7.9 in secondary care. 57\% of primary care doctors and $66 \%$ of secondary care doctors reported score of 5 or more, representing at least mild anxiety symptoms. $22 \%$ doctors in primary care and $31 \%$ doctors in secondary care reported GAD7 score of 10 or more, indicating moderate to severe anxiety. One in ten doctors in both primary and secondary care reported severe anxiety due to the ongoing COVID-19 pandemic. Conclusion. The finding of more anxiety in secondary care doctors might be because general practitioners could resort early in the pandemic to remote consultations along with inadequacy of resources, greater exposure to suffering/deaths of patients and colleagues in hospital and perceived risk of catching COVID-19 infection.

Results are limited due to relatively low numbers and it would be useful to replicate this study on a larger scale. Doctors are less likely to acknowledge their mental health difficulties due to stigma associated with mental health.

Many employers have psychological support systems in place for their staff, but it is questionable if affected individuals are willing to receive this support. This paper; therefore, calls for creating open anonymous platforms for professionals to get access to appropriate support to address their anxiety.

\section{Cardiovascular risk quantification using QRISK-3 score in people with intellectual disability}

Jamie Sin Ying $\mathrm{Ho}^{1 *}$, Vikram Rohra ${ }^{2}$, Laura Korb ${ }^{1}$ and Bhathika Perera ${ }^{1}$

${ }^{1}$ Haringey Learning Disabiliy Partnership, Barnet, Enfield an Haringey Mental Health Trust and ${ }^{2}$ North Middlesex University Hospital NHS Trust

${ }^{\star}$ Corresponding author.

doi: 10.1192/bjo.2021.187

Aims. The prevalence of cardiovascular diseases (CVD) in people with intellectual disability (ID) is around $14 \%$, higher than the general population. However, CVD risk assessments are not consistently performed. Given the high risk of premature deaths in people with ID, it is important to identify preventable risk factors and follow evidencebased interventions. QRISK-3 is a validated risk-stratification tool, which calculates the 10-year risk of developing a heart attack or stroke (https://qrisk.org/three/index.php). There are no published studies on the use of QRISK-3 in people with ID. This project aimed to 